\title{
Immediate and transient phosphorylation of the heat shock protein 27 initiates chemoresistance in prostate cancer cells
}

\author{
MATTHIAS B. STOPE ${ }^{1 *}$, MARTIN WEISS $^{1 *}$, MELANIE PREUSS $^{1}$, ANDREAS STREITBÖRGER $^{1}$, \\ CHRISTOPH A. RITTER ${ }^{2}$, UWE ZIMMERMANN ${ }^{1}$, REINHARD WALTHER $^{3}$ and MARTIN BURCHARDT $^{1}$ \\ ${ }^{1}$ Department of Urology, University Medicine Greifswald, Greifswald; ${ }^{2}$ Institute of Pharmacy, \\ Ernst-Moritz-Arndt-University of Greifswald, Greifswald; ${ }^{3}$ Department of Medical Biochemistry \\ and Molecular Biology, University Medicine Greifswald, Greifswald, Germany
}

Received June 5, 2014; Accepted July 18, 2014

DOI: $10.3892 /$ or.2014.3492

\begin{abstract}
Drug resistance minimizes the effects of prostate cancer (PC) chemotherapy with docetaxel and is generally considered to be associated with the expression of heat shock protein (HSP) 27 including various cytoprotective pathways. In the present study, we investigated the effects of HSP27 phosphorylation on PC cell growth underlying docetaxel treatment. Cell counting revealed significantly reduced cell growth during docetaxel treatment as a result of both activation of mitogen-activated protein kinase p38 (MAPK p38) and protein kinase D1 (PKD1), and, most importantly, the overexpression of the phosphorylation-mimicking mutant HSP27-3D. Further analysis revealed a docetaxel-dependent induction of HSP27 accompanied by an initial phosphorylation and rapid dephosphorylation of the protein. Based on the data, we can conclude that phosphorylation of HSP27 protein is a crucial mechanism in the initiation of chemoresistance in PC. Moreover, the results indicate a key impact of HSP27 on viability and proliferation of PC cells underlying anticancer therapy. The protective function depends on the initial phosphorylation status of HSP27 and represents a putative co-therapeutic target to prevent chemoresistance during docetaxel therapy.
\end{abstract}

\section{Introduction}

Although prostate cancer (PC) remains one of the most diagnosed malignant diseases and is the second-leading cause of tumor-associated mortality in males in the Western hemisphere (1), progression mechanisms of PC are only marginally understood. Phenotypically, it differs from a localized hormone-naive

Correspondence to: Dr Matthias B. Stope, Department of Urology, University Medicine Greifswald, Ferdinand-Sauerbruch-Strasse, D-17475 Greifswald, Germany

E-mail: matthias.stope@uni-greifswald.de

*Contributed equally

Key words: prostate cancer, HSP27, phosphorylation, docetaxel, chemoresistance state to an advanced, castration-resistant and metastatic state that is predominantly attributed to cellular proliferation in a low-level steroid hormonal environment. The first line drug docetaxel is an option for castration-resistant PC treatment; however, efficacy varies between patients and therapeutic outcome is often unsatisfying (2). Heat shock protein (HSP) 27 has been identified as controlling anti-therapeutic mechanisms by specific alterations in proliferation, cell cycle regulation, apoptosis and general stress response in cancer (3-5). In PC, it has been demonstrated that critical events in tumor progression, such as epithelial-to-mesenchymal transition, metastasis, androgen receptor (AR) signaling and treatment resistance, are promoted by HSP27 activities. Consequently, attenuation of HSP27 by newly developed inhibitors is a promising approach in the treatment of advanced PC (6). Regulation of cell physiology as well as dysregulation in malignant cells frequently depends on protein phosphorylation controlled by a coordinated network of specific kinase and phosphatase activities. HSP27 protein contains three regulatory phosphorylation sites located at the positions serine-15, -78 and -82 which are phosphorylated in the presence of diverse cellular signals, e.g. in response to oxidative and pro-inflammatory stress $(7,8)$. In PC cells, HSP27 is predominantly phosphorylated by the mitogenactivated protein kinase p38 (MAPK p38), mitogen-activated protein kinase-activated protein kinase 2 (MAPKAPK-2) pathway and by protein kinase D1 (PKD1) (9-11). Although HSP27 is estimated to orchestrate pivotal functions in PC progression and therapy, only little is known about HSP27 activity modulated by protein phosphorylation. Hassan et al described HSP27 phospho-status at serine-82 leads to suppress AR signaling in PC cells (9); however, further HSP27 functions regulated by protein phosphorylation remain unclear. Our previous studies of HSP functionality in PC progression showed HSP27 to have potent effects on AR activity $(12,13)$. Since HSP27 has been suggested to play crucial roles in the initiation and development of chemoresistance, we hypothesized that HSP27 functionality may be associated with specific protein phosphorylation/dephosphorylation. In the present study, we established a PC cell model system containing PC-3 and PC-3 cells stably overexpressing HSP27 to examine the input of HSP27 phosphorylation to HSP27-driven cytoprotective properties of docetaxel-induced chemoresistance. 


\section{Materials and methods}

Tumor cell lines and chemicals. Human epithelial PC cancer cell line PC-3 (Cell Lines Service; CLS, Eppelheim, Germany) was grown in RPMI-1640 media with $10 \%$ fetal bovine serum and $1 \%$ penicillin/streptomycin (PAN-Biotech, Aidenbach, Germany) in a $5 \% \mathrm{CO}_{2}$ atmosphere at $37^{\circ} \mathrm{C}$. PC-3-HSP27 cells stably overexpressing HSP27 were selected with $400 \mu \mathrm{g} / \mathrm{ml}$ G418 (Carl Roth, Karlsruhe, Germany) as previously described (12). For incubation experiments, docetaxel was purchased from Sigma-Aldrich (Munich, Germany), bryostatin-1 $\left(3 \times 10^{-8} \mathrm{M}\right)$ from Tocris Bioscience (Minneapolis, MN, USA), CID755673 $\left(5 \times 10^{-5} \mathrm{M}\right)$ from Axon Medchem (Groningen, Netherlands), SB203580 (10-5 M) from Selleckchem (Munich, Germany) and sorbitol $\left(3 \times 10^{-1} \mathrm{M}\right)$ was acquired from Carl Roth. Incubation with sorbitol was limited to $30 \mathrm{~min} /$ day since continuous incubation revealed cell damaging effects (14). Before the hyperosmolar shock with sorbitol, culture supernatant was removed, collected and re-added after sorbitol treatment. Drug treatment was generally initiated in adherent cells seeded $24 \mathrm{~h}$ before.

Transfection experiments. One day before transfection, cells were plated into 6-well (150,000 cells/well) or 24-well (30,000 cells/well) culture plates. For overexpression experiments, cells were transfected with $1 \mu \mathrm{g}$ DNA (24-well) and $3 \mu \mathrm{g}$ (6-well) per well, using Lipofectamine 2000 (Invitrogen, Darmstadt, Germany). The vectors pHSP27 wt, pHSP27-3A and pHSP27-3D were kindly provided by C. Kubisch (Munich, Germany) (15). pcDNA3.1 (Invitrogen) was used as empty control vector.

Western blotting. Cells were lysed in RIPA buffer [50 mM Tris (pH 7.5), $150 \mathrm{mM} \mathrm{NaCl}, 10 \mathrm{mM} \mathrm{K}_{2} \mathrm{HPO}_{4}, 5 \mathrm{mM}$ EDTA, $10 \%$ glycerol, $1 \%$ Triton $\mathrm{X}-100,0.05 \%$ sodium dodecyl sulfate, $1 \mathrm{mM} \mathrm{Na} \mathrm{VO}_{4}, 20 \mathrm{mM} \mathrm{NaF}, 0.1 \mathrm{mM}$ phenylmethylsulfonyl fluoride, $20 \mathrm{mM}$ 2-phosphoglycerate and complete protease inhibitor cocktail from Roche Applied Science; Mannheim, Germany]. Determination of protein concentration was carried out utilizing Bradford reagent (Bio-Rad) and equal amounts of protein were separated by SDS-PAGE and transferred onto a nitrocellulose membrane (Whatman, Dassel, Germany). Proteins of interest were detected by specific primary antibodies directed against HSP27, P-Ser 78 -HSP27, P-Ser 82 -HSP27, glyceraldehyde-3-phosphate dehydrogenase (GAPDH; Cell Signaling Technology, Danvers, MA, USA) and P-Ser ${ }_{15}$-HSP27 (Thermo Scientific, Waltham, MA, USA) incubated overnight and peroxidase-coupled secondary antibodies directed against mouse and rabbit (Cell Signaling Technology) incubated for $1 \mathrm{~h}$. Proteins were visualized by using SuperSignal West Dura Chemiluminescent Substrate (Thermo Scientific) and a ChemiDoc system (Bio-Rad). Quantification of protein signals was carried out by Image Lab 3.0 software (Bio-Rad).

Cell viability assay. To verify effects on cell viability, we used the 3-(4,5-dimethylthiazol-2-yl)-2,5-diphenyltetrazolium bromide (MTT) assay. For MTT assays, cells were incubated with $0.1 \mu \mathrm{g} / \mu \mathrm{l}$ aqueous MTT solution (Carl Roth) for $2 \mathrm{~h}$ at $37^{\circ} \mathrm{C}$. Formazan complexes were solubilized by the addition of $120 \mu \mathrm{l}$ dimethyl sulfoxide lysis buffer (DMSO; containing 10\%
SDS, $0.2 \% \mathrm{HCl}$ ) and dye formation was measured in a plate reader Infinite 200 PRO (Tecan, Männedorf, Switzerland) at $550 \mathrm{~nm}$ wavelength.

Cell proliferation assay. Cellular proliferation was examined by cell counting by using a CASY Cell Counter and Analyzer Model TT (Roche Applied Science). Therefore, adherent cells were detached by trypsin treatment, suspended in CASYton (Roche Applied Science) as 1:100 dilution. Measurement of $400 \mu \mathrm{l}$ cell suspension was performed in 3 cycles using a capillary of $150 \mu \mathrm{m}$ in diameter and 7.20/15.45 $\mathrm{nm}$ as gate settings to discriminate between living PC-3 and dead cells, as well as cellular debris.

Statistical analysis. Statistical comparisons of at least three independent measurements were performed using the unpaired Student's t-test with 95\% confidence interval. For all statistical analyses, results of $\mathrm{p} \leq 0.05$ were considered statistically significant. Data are provided as means \pm SD.

\section{Results}

Establishment of an in vitro cell model system for HSP27driven chemoresistance in PC. High expression of HSP27 is known to induce pro-oncogenic effects in PC (16). We used an experimental system composed of the PC cell line PC-3 and PC-3 cells stably overexpressing HSP27 (PC-3-HSP27; inset Fig. 1B) as a suitable model in which to study drug-induced and HSP27-driven effects of acquired chemoresistance. As shown by MTT assays, various concentrations of docetaxel revealed a concentration-dependent cytostatic effect in both cell lines after $72 \mathrm{~h}$ of incubation, although docetaxel sensitivity was obviously lower in PC-3-HSP27 cells for all chosen docetaxel concentrations (Fig. 1A). Overexpression of HSP27 in PC-3-HSP27 cells led to significantly increased survival rates in the presence of docetaxel at concentrations of $10^{-6} \mathrm{M}$ (1.9-fold, $\mathrm{p}=0.0258), 10^{-9} \mathrm{M}(1.8$-fold, $\mathrm{p}=0.0080)$ and $10^{-11} \mathrm{M}$ (1.3-fold, $\mathrm{p}=0.0170)$ compared to maternal PC-3 cells. These findings were validated by growth kinetics utilizing a CASY Cell Counter and Analyzer model TT for daily measurement over a period of $144 \mathrm{~h}$ (Fig. 1B). Similarly, elevated HSP27 levels resulted in enhanced cytoprotective effects during drug treatment confirmed by significantly higher survival of PC-3-HSP27 cells (48 h, 1.3-fold, p=0.0078; 72 h, 1.4-fold, $\mathrm{p}=0.0022 ; 120 \mathrm{~h}, 1.5$-fold, $\mathrm{p}=0.0392 ; 144 \mathrm{~h}, 1.4$-fold, $\mathrm{p}=0.0327$ ).

Activation of MAPK p38 and PKD1 reduce PC cell growth. MAPK p38 and PKD1 are known to be involved in important cellular regulation pathways by phosphorylation of diverse proteins, including HSP27 (9-11,17). Therefore, we assessed changes in chemosensitivity of docetaxel-treated PC-3-HSP27 cells co-incubated with modulators of MAPK p38 and PKD1 activity. Specific protein phosphorylation was activated by the use of $3 \times 10^{-1} \mathrm{M}$ sorbitol (sorb; MAPK p38) and $3 \times 10^{-8} \mathrm{M}$ bryostatin-1 (bryo; PKD1) and inhibited by the use of $10^{-5} \mathrm{M}$ SB203580 (SB; MAPK p38) and 5x10-5 M CID755673 (CID; PKD1), respectively. Studies with PC-3-HSP27 cells treated with docetaxel and exclusively incubated with a single kinase activator or inhibitor exhibited no statistically significant differences for cellular growth compared to controls (Fig. 2A). Thus, 

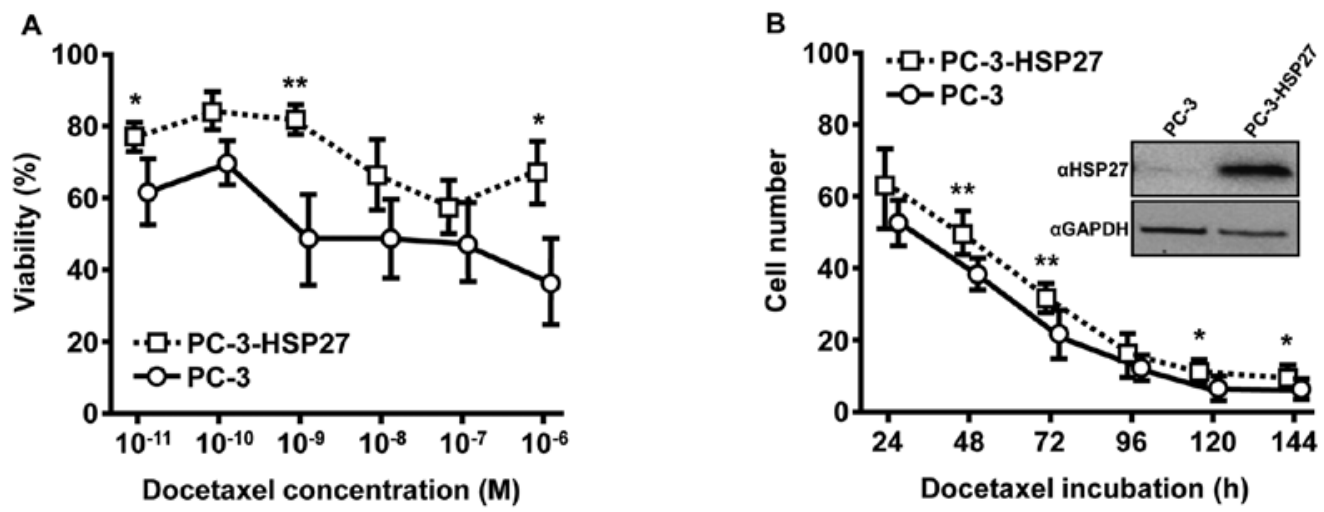

Figure 1. HSP27 overexpression confers chemoresistance to docetaxel-treated cells. (A) Cellular viability of PC-3 cells and stably HSP27 overexpressing cells PC-3-HSP27, treated with $10^{-11}$ to $10^{-6} \mathrm{M}$ docetaxel for $72 \mathrm{~h}$, were analyzed by MTT assays. Results are expressed as the means \pm SD of relative viability (docetaxel-treated cells/vehicle-treated cells). p-values were determined by Student's t-test. "p $\leq 0.05$; * $\mathrm{p} \leq 0.01$. (B) Cellular growth of PC-3 and PC-3-HSP27 cells incubated with $10^{-8} \mathrm{M}$ docetaxel over a period of $144 \mathrm{~h}$. Cells were counted at indicated time points utilizing a CASY Cell Counter and Analyzer Model TT. Results are expressed as the means \pm SD of cell count. p-values were determined by Student's t-test. " $\mathrm{p} \leq 0.05 ;{ }^{* *} \mathrm{p} \leq 0.01$. HSP27 expression levels of PC-3 compared to PC-3-HSP27 cells are shown as insert of a representative western blot detecting GAPDH as internal loading control. HSP, heat shock protein; $\mathrm{PC}$, prostate cancer.
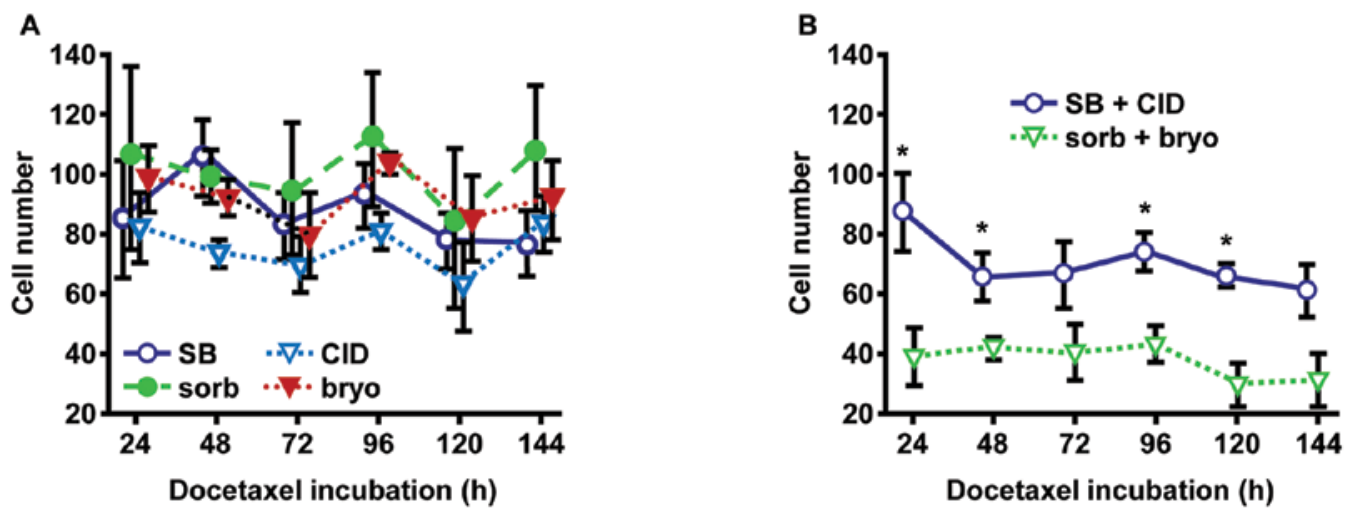

Figure 2. MAPK p38 and PKD1 suppress cell growth in prostate cancer cells stably expressing HSP27 during docetaxel treatment. (A) To study phosphorylation effects of the protein kinases MAPK p38 and PKD1 on prostate cancer cells expressing HSP27 wt protein, PC-3-HSP27 cells were incubated over a

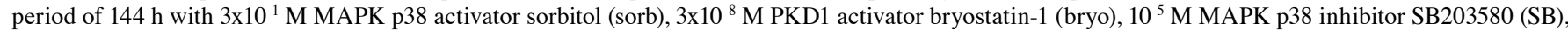
and $5 \times 10^{-5} \mathrm{M}$ PKD1 inhibitor CID755673 (CID), respectively. Cell numbers were measured at indicated time points using a CASY Cell Counter and Analyzer Model TT. Results were standardized to untreated control cells and illustrated as the means \pm SD with $p$-values determined by Student's t-test. (B) Due to negligible effects on cellular growth of cells treated with individual activators or inhibitors, the experiments were replicated applying combinations of both activators (sorb + bryo) and both inhibitors (SB + CID). Given results are indicated as the means \pm SD of cell count and cell viability, respectively, and were compared to control cells. "p $\leq 0.05$, as determined by Student's t-test. MAPK p38, mitogen-activated protein kinase p38; PKD1, protein kinase D1; HSP, heat shock protein.

we compared combinations of both activators (sorb + bryo) and both inhibitors (SB + CID). As a result, co-activated MAPK p38 and PKD1 led to increased docetaxel sensitivity of cells (Fig. 2B), measured by significantly decreased cell numbers $(24 \mathrm{~h}, 1.9$-fold, $\mathrm{p}=0.0438 ; 48 \mathrm{~h}, 1.6$-fold, $\mathrm{p}=0.0313$; $72 \mathrm{~h}, 1.6$-fold, $\mathrm{p}=0.1550 ; 96 \mathrm{~h}, 1.6$-fold, $\mathrm{p}=0.0271 ; 120 \mathrm{~h}$, 2.0 -fold, $\mathrm{p}=0.0117 ; 144 \mathrm{~h}, 2.0$-fold, $\mathrm{p}=0.1383$ ). In addition to other putative regulatory pathways, our data indicated kinasedependent control of HSP27 functions in chemoresistance, assuming higher phosphorylated HSP27 responsible for increasing sensitivity to docetaxel treatment.

HSP27-driven chemoresistance is diminished by overexpression of non-phosphorylatable HSP27-3D mutant. Considering several cellular pathways potentially being targeted by MAPK p38 and PKD1 activities and therefore being responsible for the changes in chemosensitivity, we further analyzed specific effects of HSP27 phosphorylation on chemoresistance. Cellular functionality of HSP27 depends on phosphorylation of the three regulatory phosphorylation sites, namely serine$15,-78$ and -82 (18). PC-3 cells were transiently transfected with DNA plasmids encoding for HSP27 wild-type (HSP27 wt) and HSP27 phospho-mutants: i) the non-phosphorylatable triple substitution mutant HSP27-3A (substitution of serine$15,-78$ and -82 with alanine-15, -78 and -82 ); and ii) the phosphorylation mimicking triple mutant HSP27-3D (substitution of serine-15, -78 and -82 with aspartic acid-15, -78 and -82), imitating a triple phosphorylated protein by charge and steric characteristics of the aspartic acid residues.

Transient overexpression of HSP27 wt and both HSP27 mutants (Fig. 3A) in experiments without docetaxel treatment were found to have no impact on cellular growth (Fig. 3B). The 
A
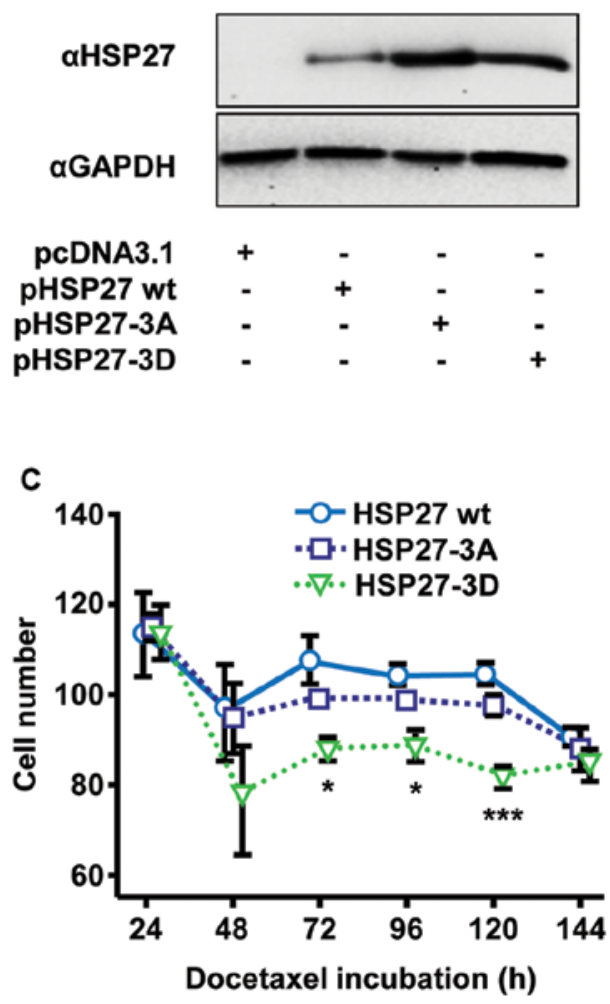

B

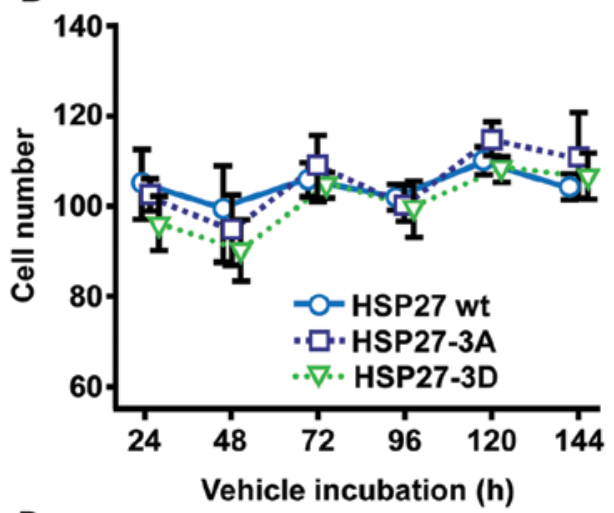

D

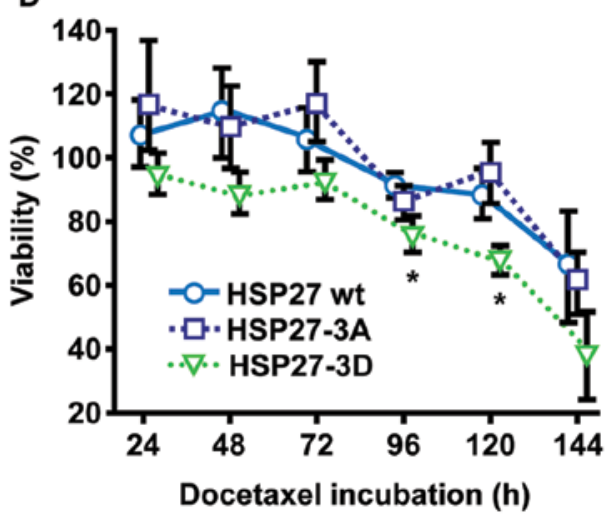

Figure 3. Permanently phosphorylated HSP27 diminishes the efficiency of HSP27-mediated cytoprotection in the presence of docetaxel. (A) Overexpression of HSP27 wt and mutant proteins visualized by western blot analysis $48 \mathrm{~h}$ after transfection. The representative blot was incubated with an antibody specific to total HSP27 in reference to GAPDH. The graphs show results as means \pm SD. p-values were determined by Student's t-test. (B) The influence of transiently overexpressed HSP27 wt and mutant proteins HSP27-3A and HSP27-3D to growth of PC-3 cells were measured by cell counting (CASY Cell Counter and Analyzer Model TT) in the absence of docetaxel over a period of $144 \mathrm{~h}$. The graphs illustrate results as means \pm SD and p-values determined by Student's t-test. (C) Cell count and (D) cell viability (MTT) were determined at indicated time points to assess the putative roles of HSP27 wt, the non-phosphorylatable triple substitution mutant HSP27-3A, and the phosphorylation-mimicking triple mutant HSP27-3D. All data were standardized to vehicle-transfected control cells, which are defined as $100 \%$. Results are presented as means \pm SD. ${ }^{*} \mathrm{p} \leq 0.05 ;{ }^{* * *} \mathrm{p} \leq 0.001$, as determined by Student's t-test. HSP, heat shock protein; PC, prostate cancer.

incubation with docetaxel, however, caused notable reductions in proliferation of PC-3 cells overexpressing phosphorylation mimicking HSP27-3D compared to cells overexpressing HSP27 wt and non-phosphorylatable HSP27-3A (Fig. 3C). Compared to HSP27 wt and HSP27-3A, respectively, HSP27-3D-expressing cells exhibited significantly reduced cell proliferation $(72 \mathrm{~h}, 1.2$-fold reduction, $\mathrm{p}=0.0329 ; 96 \mathrm{~h}, 1.2$-fold reduction, $\mathrm{p}=0.0226 ; 120 \mathrm{~h}, 1.3$-fold reduction, $\mathrm{p}=0.0005)$. As expected, similar findings were noted by further analyses of cellular viability using MTT assay (Fig. 3D). Again, HSP27-3D revealed poorer cellular viability of transfected, docetaxel-treated cells, when comparing overexpression of HSP27-3D with HSP27 wt and HSP27-3A (96 h, 1.2-fold reduction, $\mathrm{p}=0.0415 ; 120 \mathrm{~h}, 1.3$-fold reduction, $\mathrm{p}=0.0087$ ). Analysis of cell counting (Fig. 3C) as well as cellular viability (Fig. 3D) of HSP27 wt- and HSP27-3A-transfected cells showed no differences.

Docetaxel causes rapid and transient phosphorylations of HSP27. To clarify how HSP27-driven chemoresistance is activated and regulated throughout the treatment with docetaxel, we analyzed changes in the phosphorylation status of HSP27 utilizing phospho-specific HSP27 antibodies (Fig.4A,C andE). Vehicle-treated control cells did not obviously change their status of low-phosphorylated HSP27 (Fig. 4B, $\mathrm{D}$ and $\mathrm{F}$ ). In comparison, protein phosphorylation in the presence of docetaxel was significantly increased after $24 \mathrm{~h}$ at HSP27 phosphorylation sites serine-15 (9.4-fold, $\mathrm{p}=0.0071)$ and serine-82 (12.6-fold, $\mathrm{p}=0.0150)$. Analysis of serine-78 phosphorylation revealed similar results. However, statistical assessment procedure failed to reach significance. Notably, the strong induction of HSP27 phosphorylation was largely reversed after $48 \mathrm{~h}$ of docetaxel incubation and remained constantly low for $144 \mathrm{~h}$ of observation. Periods of incubation $>24 \mathrm{~h}$ did not differ in HSP27 phosphorylation between vehicle- and docetaxel-treated groups (Fig. 4B, D and F).

\section{Discussion}

In the present study, we demonstrated that treatment of PC cells with docetaxel, a first-line therapy drug for castration-resistant PC, induces phosphorylation of HSP27, which is a cytoprotective factor counter-acting antiproliferative cancer therapies (18). Markedly, we detected a rapid phosphorylation within the first day of docetaxel incubation at all of the three regulatory phosphorylation sites immediately followed by dephosphorylation. Furthermore, permanently phosphorylated HSP27 was associated with attenuated cytoprotective proper- 
A

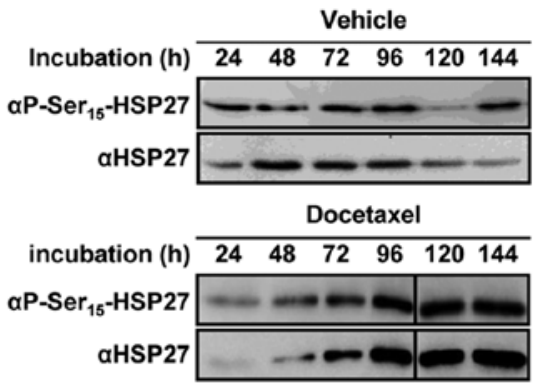

C

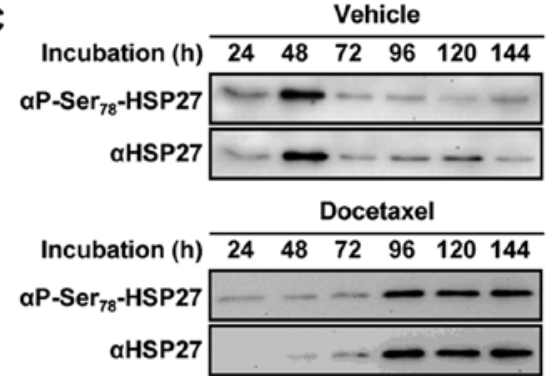

E

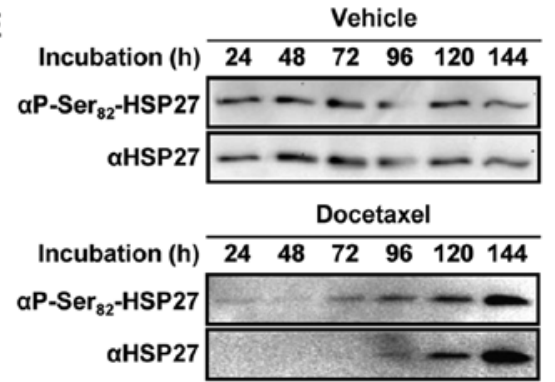

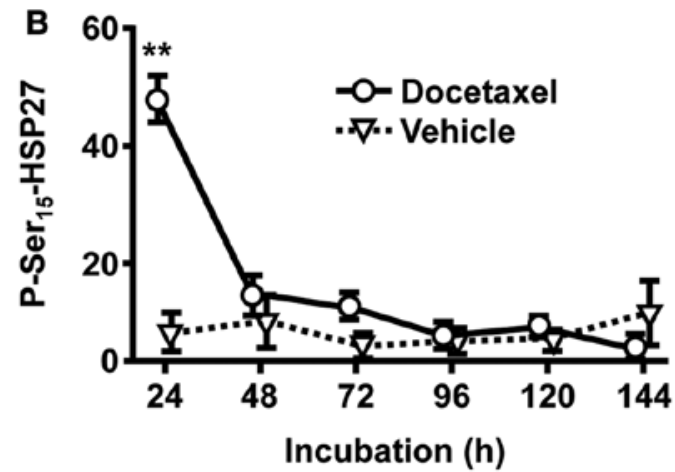
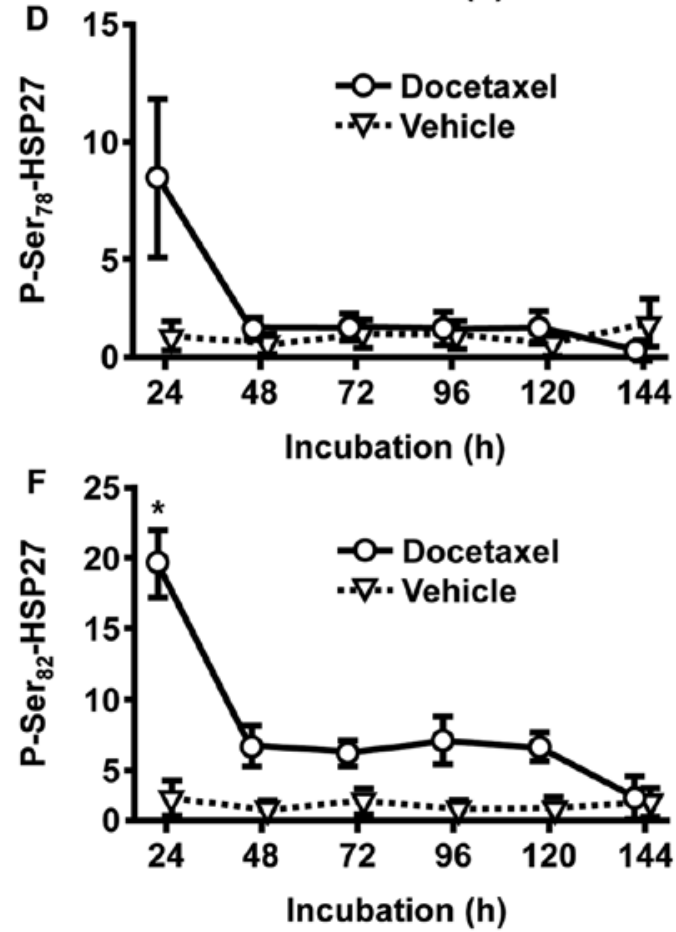

Figure 4. Phosphorylation of HSP27 is significantly induced after docetaxel treatment. (A-F) Elevation of HSP27 phosphorylation consecutive to docetaxel treatment declined within $24 \mathrm{~h}$ of incubation and remained at a low level. PC-3 cells transiently transfected with HSP27 wt were treated with $10 \mathrm{nM}$ docetaxel to analyze changes of HSP27 phosphorylation at the three regulatory phosphorylation sites serine-15, -78 and -82 . Cells were harvested after indicated time points of docetaxel incubation and subjected to western blot analysis utilizing the phospho-specific antibodies P-Ser15-HSP27, P-Ser78-HSP27 and P-Ser82-HSP27. Site-specific phosphorylation was standardized to the total amount of HSP27 and GAPDH served as loading control. Representative western blot analyses are shown (A, C and E). Graphs of vehicle- and docetaxel-treated samples were calculated as the means \pm SD of ratio of phosphorylated HSP27 to total HSP27 (B, D and F). p-values were determined by Student's t-test. ${ }^{*} \mathrm{p} \leq 0.05,{ }^{* *} \mathrm{p} \leq 0.01$. HSP, heat shock protein; PC, prostate cancer.

ties, strongly supporting the theory that rapid but short-termed phosphorylation of HSP27 is an important step in HSP27driven initiation of chemoresistance.

HSP27 induction has been detected in various solid tumors. Hence, HSP27 may be regarded as a pivotal factor for progression and treatment resistance. In PC cells, HSP27 has a crucial role in tumor-specific and therapy-induced cytoprotection enabling tumor growth in the setting of hormonal ablation and cytostatic therapy. It is well recognized that HSP27 properties control activities of various signaling molecules, such as $\beta$-catenin, E-cadherin, interleukins, transforming growth factor $\beta$ and AR, with all these effector proteins frequently dysregulated in PC and shown to exert oncogenic transformation (12,19-22). As shown in Fig. 1, stable overexpression of HSP27 in PC-3 cells revealed significantly enhanced resistance to docetaxel treatment in a concentration- and time-dependent manner. These findings, in accordance with previous studies (14), reflect HSP27-driven cytoprotectivity in PC.
Biological functions of HSP27 are mainly regulated by protein phosphorylation, suggesting that HSP27 phosphorylation may concur with chemoresistance mechanisms in PC cells. Therefore, it is reasonable to assume that temporal and spatial phosphorylation/dephosphorylation of HSP27 by kinase and phosphatase activities are an essential part of HSP27 in the regulation of treatment-resistant processes.

It has been reported that kinase pathways of MAPK p38 and PKD1 are major regulators of HSP27 properties in PC $(9,23)$. Since we showed that the pharmacological activation of MAPK p38 and PKD1 kinases led to elevated docetaxel sensitivity (Fig. 2B), it is conceivable that this sensitizing effect, at least to some extent, is mediated through HSP27 phosphorylation. Accordingly, HSP27 phosphorylation by MAPK p38 and PKD1 was already found in PC and other cancer entities $(9-11,16)$. However, due to both kinases operating in numerous signaling pathways, we cannot exclude the possibility that both kinases affect additional cellular targets whose modulation may 
contribute to enhanced antiproliferative effects in the presence of docetaxel. To examine this possibility, we focused on transfection experiments utilizing DNA plasmids encoding for the well-approved HSP27 mutants HSP27-3A and HSP27-3D representing an unphosphorylatable and a phospho-mimicking type of HSP27, respectively. Expression of HSP27-3A obtained no alteration on docetaxel sensitivity whereas expression of HSP27-3D resulted in significantly increased sensitivity to docetaxel (Fig. 3C and D). These observations were supported by western blot analysis using an experimental chemotherapy model which exhibited rapid and transient phosphorylations of HSP27, a mechanism similar to HSP27-driven drug resistance described in breast and pancreatic cancer cells $(24,25)$. In particular, we detected docetaxel-induced phosphorylation of HSP27 amino acids serine- $15,-78$ and -82 after $24 \mathrm{~h}$ of incubation (Fig. 4). Experiments in high time resolution (1-24 h) exhibited a rapid but transient HSP27 phosphorylation within the first $8 \mathrm{~h}$ (data not shown).

Although our experiments clearly indicate that permanently phosphorylated HSP27 increases chemosensitivity, our current understanding of HSP27 phospho-regulation is still limited. Nakashima et al showed enhanced chemosensitivity in gemcitabine-treated pancreatic cancer cells with phosphorylated HSP27 protein (25), confirming our own observations. In contrast, Taba et al reported converse effects of phosphorylated HSP27 in pancreatic cancer cells in the presence of gemcitabine, which was confirmed in a cell model system of 5-fluorouracil-resistant colon cancer (26,27). Furthermore, some studies provide examples for growth regulatory properties of phosphor-HSP27 in the absence of chemotherapeutics $(28,29)$. Collectively, regulation of HSP27 appears to be part of a complex regulatory network controlled by kinases and phosphatases and may be induced in a drug- and cell typespecific manner.

Protein phosphorylation at multiple sites is often catalyzed in a fixed order. However, human HSP27 phosphorylation does not occur in an obligatory sequence (11). Although HSP27 phosphorylation has been shown in response to various stimuli, such as cytokines, receptor ligands, glucose and metals (18), to our knowledge this study is the first to report HSP27 phosphorylation initiated by drug treatment of PC cells. Due to the transient character of the HSP27 phosphorylation which lasts several hours, strongly orchestrated activities of kinases and phosphatases are required. According to our observations and the data from previous studies, most probably MAPK p38 and PKD1 pathways exert HSP27 phosphorylation (18). Since several kinases were described using HSP27 as substrate for protein phosphorylation, e.g. various MAPK-activated kinases and protein kinase $\mathrm{C}$ (30-32), it cannot be excluded that further kinase pathways contribute to HSP27 phosphorylation. Despite the increase in molecular insights into HSP27 phosphorylation in cancer mechanisms, functions of HSP27 dephosphorylation are for the most part unknown. Several studies have shown that inhibition of protein phosphatase 2A (PP2A) affects the phospho-status of human HSP27 $(33,34)$. Notably, Liu et al recently described that dual specificity protein phosphatase 1 (DUSP1) controls treatment resistance in pancreatic cancer by affecting MAPK pathways (35), thus, it is consistent with our observations indicating potential targets for further examinations.
In conclusion, our findings enhance our understanding of survival mechanisms in drug-resistant PC cells and qualify HSP27 phosphorylation pathways as appropriate targets for new anticancer strategies. Future research will clarify if kinase activators as well as phosphatase inhibitors are a potent opportunity to support existing HSP27-targeting anticancer therapies.

\section{Acknowledgements}

This study was supported in part by a grant from the Dr Robert Pfleger-Foundation, Bamberg, Germany. The authors thank Anne Brandenburg and Katja Wittig for their technical assistance.

\section{References}

1. Jemal A, Siegel R, Ward E, Hao Y, Xu J, Murray T and Thun MJ: Cancer statistics, 2008. CA Cancer J Clin 58: 71-96, 2008.

2. Hwang C: Overcoming docetaxel resistance in prostate cancer: a perspective review. Ther Adv Med Oncol 4: 329-340, 2012.

3. Beere HM, Wolf BB, Cain K, Mosser DD, Mahboubi A, Kuwana T, Tailor P, Morimoto RI, Cohen GM and Green DR: Heat-shock protein 70 inhibits apoptosis by preventing recruitment of procaspase-9 to the Apaf-1 apoptosome. Nat Cell Biol 2: $469-475,2000$

4. Haslbeck M and Buchner J: Chaperone function of sHsps. Prog Mol Subcell Biol 28: 37-59, 2002.

5. Parcellier A, Brunet M, Schmitt E, Col E, Didelot C, Hammann A, Nakayama K, Nakayama KI, Khochbin S, Solary E and Garrido C: HSP27 favors ubiquitination and proteasomal degradation of $27^{\mathrm{Kipl}}$ and helps S-phase re-entry in stressed cells. FASEB J 20: 1179-1181, 2006.

6. Ischia J, Saad F and Gleave M: The promise of heat shock protein inhibitors in the treatment of castration resistant prostate cancer. Curr Opin Urol 23: 194-200, 2013.

7. Liu T, Guevara OE, Warburton RR, Hill NS, Gaestel M and Kayyali US: Modulation of HSP27 alters hypoxia-induced endothelial permeability and related signaling pathways. J Cell Physiol 220: 600-610, 2009.

8. Tsuji F, Oh-hashi K and Kiuchi K: Differential effects of Akt pathway inhibitors on IL-1 $\beta$-induced protein phosphorylation in human fibroblast-like synoviocytes. J Recept Signal Transduct Res 32: 22-28, 2012.

9. Hassan S, Biswas MH, Zhang C, Du C and Balaji KC: Heat shock protein 27 mediates repression of androgen receptor function by protein kinase D1 in prostate cancer cells. Oncogene 28: 4386-4396, 2009.

10. Evans IM, Britton G and Zachary IC: Vascular endothelial growth factor induces heat shock protein (HSP) 27 serine 82 phosphorylation and endothelial tubulogenesis via protein kinase D and independent of p38 kinase. Cell Signal 20: 1375-1384, 2008.

11. Landry J, Lambert H, Zhou M, Lavoie JN, Hickey E, Weber LA and Anderson CW: Human HSP27 is phosphorylated at serines 78 and 82 by heat shock and mitogen-activated kinases that recognize the same amino acid motif as S6 kinase II. J Biol Chem 267: 794-803, 1992.

12. Stope MB, Schubert T, Staar D, Rönnau C, Streitbörger A, Kroeger N, Kubisch C, Zimmermann U, Walther R and Burchardt M: Effect of the heat shock protein HSP27 on androgen receptor expression and function in prostate cancer cells. World $\mathbf{J}$ Urol 30: 327-331, 2012.

13. Stope MB, Bradl J, Peters S, Streitbörger A, Weiss M, Zimmermann U, Walther R, Lillig $\mathrm{CH}$ and Burchardt $\mathrm{M}$ : Shortened isoforms of the androgen receptor are regulated by the cytoprotective heat-shock protein HSPB1 and the tumor-suppressive microRNA miR-1 in prostate cancer cells. Anticancer Res 33: 4921-4926, 2013.

14. Dokas LA, Malone AM, Williams FE, Nauli SM and Messer WS Jr: Multiple protein kinases determine the phosphorylated state of the small heat shock protein, HSP27, in SH-SY5Y neuroblastoma cells. Neuropharmacology 61: 12-24, 2011.

15. Kubisch C, Dimagno MJ, Tietz AB, Welsh MJ, Ernst SA, BrandtNedelev B, Diebold J, Wagner AC, Göke B, Williams JA and Schäfer C: Overexpression of heat shock protein Hsp27 protects against cerulein-induced pancreatitis. Gastroenterology 127 : 275-286, 2004 
16. Rocchi P, So A, Kojima S, Signaevsky M, Beraldi E, Fazli L, Hurtado-Coll A, Yamanaka K and Gleave M: Heat shock protein 27 increases after androgen ablation and plays a cytoprotective role in hormone-refractory prostate cancer. Cancer Res 64: 6595-6602, 2004.

17. Cuenda A, Rouse J, Doza YN, Meier R, Cohen P, Gallagher TF, Young PR and Lee JC: SB 203580 is a specific inhibitor of a MAP kinase homologue which is stimulated by cellular stresses and interleukin-1. FEBS Lett 364: 229-233, 1995.

18. Kostenko S and Moens U: Heat shock protein 27 phosphorylation: kinases, phosphatases, functions and pathology. Cell Mol Life Sci 66: 3289-3307, 2009.

19. Jaggi M, Rao PS, Smith DJ, Hemstreet GP and Balaji KC: Protein kinase $\mathrm{C}$ mu is down-regulated in androgen-independent prostate cancer. Biochem Biophys Res Commun 307: 254-260, 2003.

20. Mak P, Jaggi M, Syed V, Chauhan SC, Hassan S, Biswas H and Balaji KC: Protein kinase D1 (PKD1) influences androgen receptor (AR) function in prostate cancer cells. Biochem Biophys Res Commun 373: 618-623, 2008.

21. Syed V, Mak P, Du C and Balaji KC: $\beta$-catenin mediates alteration in cell proliferation, motility and invasion of prostate cancer cells by differential expression of E-cadherin and protein kinase D1. J Cell Biochem 104: 82-95, 2008.

22. Di K, Wong YC and Wang X: Id-1 promotes TGF- $\beta 1$-induced cell motility through HSP27 activation and disassembly of adherens junction in prostate epithelial cells. Exp Cell Res 313: 3983-3999, 2007.

23. Zoubeidi A, Zardan A, Beraldi E, Fazli L, Sowery R, Rennie P, Nelson $\mathrm{C}$ and Gleave $\mathrm{M}$ : Cooperative interactions between androgen receptor (AR) and heat-shock protein 27 facilitate AR transcriptional activity. Cancer Res 67: 10455-10465, 2007.

24. Xu Y, Diao Y, Qi S, Pan X, Wang Q, Xin Y, Cao X, Ruan J, Zhao Z, Luo L, Liu C and Yin Z: Phosphorylated Hsp27 activates ATM-dependent p53 signaling and mediates the resistance of MCF-7 cells to doxorubicin-induced apoptosis. Cell Signal 25: 1176-1185, 2013

25. Nakashima M, Adachi S, Yasuda I, Yamauchi T, Kawaguchi J, Itani M, Yoshioka T, Matsushima-Nishiwaki R, Hirose Y, Kozawa $\mathrm{O}$ and Moriwaki H: Phosphorylation status of heat shock protein 27 plays a key role in gemcitabine-induced apoptosis of pancreatic cancer cells. Cancer Lett 313: 218-225, 2011.

26. Taba K, Kuramitsu Y, Ryozawa S, Yoshida K, Tanaka T, Maehara S, Maehara Y, Sakaida I and Nakamura K: Heat-shock protein 27 is phosphorylated in gemcitabine-resistant pancreatic cancer cells. Anticancer Res 30: 2539-2543, 2010.
27. Sakai A, Otani M, Miyamoto A, Yoshida H, Furuya E and Tanigawa N: Identification of phosphorylated serine-15 and -82 residues of HSPB1 in 5-fluorouracil-resistant colorectal cancer cells by proteomics. J Proteomics 75: 806-818, 2012.

28. Mouratidis PX, Colston KW, Bartlett JB, Muller GW, Man HW, Stirling D and Dalgleish AG: Antiproliferative effects of CC-8062 and CC-8075 in pancreatic cancer cells. Pancreas 38: 78-84, 2009.

29. Garrido C, Ottavi P, Fromentin A, Hammann A, Arrigo AP, Chauffert B and Mehlen P: HSP27 as a mediator of confluencedependent resistance to cell death induced by anticancer drugs. Cancer Res 57: 2661-2667, 1997.

30. Maizels ET, Peters CA, Kline M, Cutler RE Jr, Shanmugam M and Hunzicker-Dunn M: Heat-schock protein-25/27 phosphorylation by the $\mathrm{d}$ isoform of protein kinase C. Biochem J 332: 703-712, 1998.

31. Clifton AD, Young PR and Cohen P: A comparison of the substrate specificity of MAPKAP kinase-2 and MAPKAPkinase- 3 and their activation by cytokines and cellular stress. FEBS Lett 392: 209-214, 1996

32. Guo K, Liu Y, Zhou H, Dai Z, Zhang J, Sun R, Chen J, Sun Q, $\mathrm{Lu} \mathrm{W}$, Kang $\mathrm{X}$ and Chen P: Involvement of protein kinase $\mathrm{C}$ $\beta$-extracellular signal-regulating kinase ${ }_{12} / \mathrm{p} 38$ mitogen-activated protein kinase-heat shock protein 27 activation in hepatocellular carcinoma cell motility and invasion. Cancer Sci 99: 486-496, 2008.

33. Suga H, Nakajima K, Shu E, Kanno Y, Hirade K, Ishisaki A, Matsuno H, Tanabe K, Takai S, Akamatsu S, Kato K, Oiso Y and Kozawa O: Possible involvement of phosphatidylinositol 3-kinase/Akt signal pathway in vasopressin-induced HSP27 phosphorylation in aortic smooth muscle A10 cells. Arch Biochem Biophys 438: 137-145, 2005.

34. Yuan J and Rozengurt E: PKD, PKD2, and p38 MAPK mediate Hsp27 serine-82 phosphorylation induced by neurotensin in pancreatic cancer PANC-1 cells. J Cell Biochem 103: 648-662, 2008.

35. Liu F, Gore AJ, Wilson JL and Korc M: DUSP1 is a novel target for enhancing pancreatic cancer cell sensitivity to gemcitabine. PLoS One 9: e84982, 2014. 